\title{
CONSUMO MÁXIMO DE OXÍGENO (VO2max) EN BOMBEROS: REVISIÓN SISTEMÁTICA DE ESTUDIOS
}

\author{
Jimmy Rojas Quirós \\ Escuela de Ciencias del Movimiento Humano y Calidad de Vida. \\ Facultad de Ciencias de la Salud, Universidad Nacional de Costa \\ Rica. \\ jiroqui@gmail.com
}

\begin{abstract}
RESUMEN
Consumo máximo de oxígeno (VO2max) en bomberos: revisión sistemática de estudios. El trabajo de bombero es catalogada como una profesión de riesgo, con alto requerimiento físico; en los últimos años el bombero ha adquirido más competencias que la propia de apagar incendios, entre ellas las intervenciones en accidentes de tránsito, incidentes con materiales peligrosos, rescates, fuegos forestales, entre otros. La imagen del bombero tradicional ha dado paso a nuevos profesionales preparados para afrontar todo tipo de desastres por lo que se habla en la actualidad del bombero completo. El objetivo de este estudio fue revisar sistemáticamente la evidencia científica sobre los valores de consumo máximo de oxígeno (VO2max) que presentan los bomberos. Se incluyeron un total de 19 estudios, publicados entre los años 2002 y 2012, de las bases de datos de EBSCOhost, SpringerLink, SPORTDiscus, Medline, Embase y Scielo; además de revistas como Journal of Sports Science \& Medicine, Occupational Medicine y Psicothema. Se concluye con esta revisión, con base a los resultados de los estudios, que las diferentes poblaciones de bomberos, oscilan un VO2max de 39,20 a 58,21 $\mathrm{ml} / \mathrm{kg} / \mathrm{min}$, que varía según la condición inicial que tienen los bomberos en los diferentes estudios que proponen los autores; en general se determina que un bombero debe poseer valores de VO2max por encima de los $43 \mathrm{ml} / \mathrm{kg} / \mathrm{min}$, lo cual es lo mínimo recomendable para al menos poseer una buena capacidad aeróbica que le permita desenvolverse en sus funciones básicas como bombero.
\end{abstract}

PALABRAS CLAVE: Bomberos, consumo de oxígeno, entrenamiento, capacidades físicas, ejercicio, capacidad aeróbica

\begin{abstract}
Maximal oxygen consumption (VO2max) in firefighters: a systematic review of studies. The job of a firefighter is listed as a risk profession with high physical requirement, and in recent years the firefighter has acquired more powers itself out fires, including interventions in traffic accidents, hazardous materials incidents, rescues, forest fires, among others. The traditional firefighter image has given way to new professionals ready to face all kinds of disasters by the talk in full firefighter today. The aim of this study was to systematically review the scientific evidence on the values of maximum oxygen consumption (VO2max) having firefighters. We included a total of 19 studies, published between 2002 and 2012, from EBSCOhost databases, SpringerLink, SPORTDiscus, Medline, Embase and SciELO, besides journals as Journal of Sports Science \& Medicine, Occupational Medicine and Psicothema. We conclude this review, based on the results of the studies, that different populations of fire, one VO2max ranging from 39.20 to $58.21 \mathrm{ml} / \mathrm{kg} / \mathrm{min}$, which varies according to the initial condition with firefighters different studies proposed by the authors, generally it is determined that a firefighter must have VO2max values above $43 \mathrm{ml} / \mathrm{kg} / \mathrm{min}$, which is the minimum recommended to at least have a good aerobic capacity that allows function in its core functions as a firefighter.
\end{abstract}

KEY WORDS: Firefighters, oxygen consumption, training, physical abilities exercise, aerobic capacity 


\section{INTRODUCCIÓN}

Desde los procesos de inducción los aspirantes a pertenecer al cuerpo de bomberos deben someterse a fases de entrenamiento para mejorar sus capacidades físicas, incluida la capacidad cardiorrespiratoria; el aumentar el rendimiento físico le será de gran utilidad para su práctica profesional dentro de sus labores como bomberos (Lara, García, Torres y Zagalaz, 2012).

El bombero debe mantener una buena condición física a lo largo de toda su vida laboral, ya que su actividad implica actuaciones que así lo requieren, realizando esfuerzos físicos importantes (Lara et al., 2012; Ares, 2008; Saborit et al., 2010). El que un bombero se encuentre en buena forma física va a retrasar la fatiga, lo que es muy positivo para su labor profesional que incluye constantemente levantar objetos pesados, subir escaleras, gatear o estar de pie por largos periodos de tiempo; no se puede evitar que en la rutina diaria de los bomberos estos lleguen a estar cansados tanto físicamente como emocionalmente (Rivera y Rivera, 2009), pero un bombero que practique ejercicios físicos estará mejor preparado para esas situaciones.

La capacidad aeróbica está en función del volumen máximo de oxígeno $\left(\mathrm{VO}_{2} \mathrm{máx}\right)$ el cual representa la capacidad máxima del organismo para metabolizar el oxígeno en la sangre (Saborit et al., 2010). A un mayor consumo de oxígeno por parte de los bomberos son mayores las posibilidades no sólo de resistir a las demandas físicas a las cuales son sometidos, sino también a disminuir el riesgo de padecer enfermedades cardiorrespiratorias provocadas por el constante contacto con el humo y gases tóxicos.

$\mathrm{El} \mathrm{VO}_{2} \max$ es la mejor manera de medir la resistencia cardiorrespiratoria (Wilmore y Costill, 2007); en personas sedentarias los valores normales se encuentran entre 35 a 42 $\mathrm{ml} / \mathrm{kg} / \mathrm{min}$ (Wilmore y Costill, 2007); mientras que López y Fernández (2006) definen el $\mathrm{VO}_{2}$ max como "la cantidad máxima de oxígeno que el organismo es capaz de absorber, transportar y consumir por unidad de tiempo" (p. 409); estos autores localizan los valores normales para hombres y mujeres de entre 20 a 40 años en 35 a $45 \mathrm{ml} / \mathrm{kg} / \mathrm{min}$ y de 30 a 40 $\mathrm{ml} / \mathrm{Kg} / \mathrm{min}$, respectivamente.

El bombero se encuentra constantemente en riesgo y este se aumentará si su condición física no es adecuada, no sólo por la incapacidad del organismo de controlar todas las alteraciones fisiológicas al romper la homeostasis en una emergencia donde el bombero debe actuar, sino, además en el lugar del incidente donde deba esquivar obstáculos, saltar o inclusive correr, y sus posibilidades físicas no se lo permitan. Las principales causas de atención que se presentaron los bomberos en Estados Unidos desde 1990 al 2008, se encuentran los problemas cardiacos, las quemaduras y las intoxicaciones, siendo los problemas cardiacos la principal causa (Smith; 2011).

Kales et al. (cit. por Rodríguez et al., 2007) enfocaron la importancia de lograr y mantener una buena condición física a la hora de disminuir el riesgo de enfermedades cardiovasculares y reducir la mortalidad en bomberos; en la investigación de Kales et al. establecieron que durante el periodo comprendido entre los años 1994 y 2004, 
aproximadamente el $32 \%$ de las muertes por fallo cardiaco en bomberos se produjeron en el momento que se encontraban apagando un incendio.

Las tres causas principales de riesgo de accidente de trabajo en este colectivo son, por orden decreciente, los sobreesfuerzos y posturas forzadas, la falta de formación e información, y el cansancio o fatiga (Ares, 2008).

En el momento de las emergencias, al acudir a la extinción de los incendios, esta labor se convierte en una ocupación aeróbicamente exigente. Estas situaciones de emergencia requieren que un bombero deba trabajar en o cerca de su máxima capacidad durante varios minutos (Mier y Gibson, 2004).

Ante las altas exigencias físicas en las emergencias en los incendios o desastres, se necesita tener un alto desempeño en el trabajo, pero frente a la gran exigencia también se eleva las posibilidades de una lesión; para minimizar estas posibilidades de una lesión se dependerá en gran medida del nivel de condición física de los bomberos (Mier y Gibson, 2004).

Kianmehr y Nazem (2011) indicaron que el coste de oxígeno de un bombero es de $60 \%$ a $80 \%$ de su importe máximo durante el rescate y auxilio de la operación. Además, el personal inactivo tiene $90 \%$ o más la posibilidad de tener enfermedades cardiovasculares en comparación con las personas que tienen condición física óptima (Mier y Gibson, 2004).

Es fundamental integrar los múltiples estudios relacionados a las capacidades físicas de los bomberos, específicamente al $\mathrm{VO}_{2}$ max para que de forma sistemática se puedan generar conclusiones generales que permitan establecer los niveles de $\mathrm{VO}_{2}$ max que al menos deben tener los dedicados a esta profesión; es por esa razón la importancia de este tipo de estudio, que permitirá conocer las diversas investigaciones que enfocan sobre el $\mathrm{VO}_{2}$ max de los bomberos, como una de las capacidades condicionantes fundamentales en los procesos de preparación física; y así, los preparadores físicos pueden establecer las cargas de entrenamiento con mayor eficiencia para esta población.

El propósito del estudio fue determinar los niveles de $\mathrm{VO}_{2}$ max encontrados en los estudios en poblaciones de bomberos, para poder ejercer con tranquilidad y con eficiencia su labor diaria, con base en una revisión sistemática de literatura.

\section{METODOLOGÍA}

Búsqueda de literatura. Se realizó una búsqueda electrónica de artículos científicos en idioma inglés y español, publicados entre los años 2002 y 2012, provenientes de las bases de datos de EBSCOhost, SpringerLink, SPORTDiscus, Medline, Embase y Scielo; además de revistas como Journal of Sports Science \& Medicine, Occupational Medicine y Psicothema. Se utilizaron las siguientes palabras clave para la revisión: bomberos, consumo de oxígeno, entrenamiento, capacidades físicas, ejercicio. Los estudios que se incluyeron se limitan a reportes completos de investigaciones que informen sobre el consumo de oxígeno $\left(\mathrm{VO}_{2}\right)$ y el $\mathrm{VO}_{2} \max$ que tienen los bomberos. Se incluyeron como parámetros de la búsqueda estudios experimentales puros, estudios descriptivos y revisiones sistemáticas. 
Se encontraron 40 artículos que fueron analizados en la revisión, pero solo se seleccionaron 19 que cumplieron con los criterios establecidos anteriormente, esto después de revisar los títulos, leer los resúmenes correspondientes y analizar la información propuesta por los autores.

\section{RESULTADOS Y DISCUSIÓN}

En la variedad de estudios analizados se presentan los valores del $\mathrm{VO}_{2}$ max encontrados en poblaciones de bomberos, en la tabla 1 se muestran las características generales de estos estudios.

Tabla 1

Características generales de los estudios incluidos en esta revisión sistemática.

\begin{tabular}{|c|c|c|}
\hline Autores & Año / País & Temática \\
\hline Mier y Gibson & $\begin{array}{c}2004 \\
\text { Estados Unidos }\end{array}$ & $\begin{array}{l}\text { Predicen a través de una prueba de esfuerzo la } \\
\text { capacidad aeróbica de los bomberos }\end{array}$ \\
\hline Mendenhall et al. & $\begin{array}{c}2005 \\
\text { Estados Unidos }\end{array}$ & $\begin{array}{l}\text { Su objetivo es validar una evaluación de trabajo } \\
\text { rendimiento físico para bomberos }\end{array}$ \\
\hline Rodríguez et al. & $\begin{array}{c}2007 \\
\text { España }\end{array}$ & $\begin{array}{l}\text { Analizan la intensidad de esfuerzo alcanzada por } \\
\text { el personal especialista en extinción de incendios } \\
\text { forestales durante sus labores. }\end{array}$ \\
\hline Dreger y Petersen & $\begin{array}{l}2007 \\
\text { Canadá }\end{array}$ & $\begin{array}{l}\text { Analizan resultados de oxigenación obtenidas en } \\
\text { la aplicación de test de salud para bomberos, } \\
\text { tanto hombres como mujeres. }\end{array}$ \\
\hline Satué et al. & $\begin{array}{c}2007 \\
\text { España }\end{array}$ & $\begin{array}{l}\text { Estudian los factores condicionantes del } \\
\text { rendimiento físico de los bomberos forestales en } \\
\text { las pruebas de aptitud física, para la selección del } \\
\text { personal. }\end{array}$ \\
\hline Villa et al. & $\begin{array}{c}2009 \\
\text { España }\end{array}$ & $\begin{array}{l}\text { Analizan la intensidad de esfuerzo alcanzada por } \\
\text { el personal especialista en extinción de incendios } \\
\text { forestales. }\end{array}$ \\
\hline Rojas, J. & $\begin{array}{c}2009 \\
\text { Costa Rica }\end{array}$ & $\begin{array}{l}\text { Informa sobre el proceso de preparación física de } \\
\text { los bomberos en inducción en Costa Rica. }\end{array}$ \\
\hline Rivera y Rivera & $\begin{array}{l}2009 \\
\text { Ecuador }\end{array}$ & $\begin{array}{l}\text { Estudian el consumo máximo de oxígeno, el } \\
\text { sedentarismo y la carga de trabajo físico en } \\
\text { bomberos de Ecuador. }\end{array}$ \\
\hline
\end{tabular}


Donovan et al.

2009

Estados Unidos

Saborit et al.

Drew-Nord et al.

Smith, D

Nazem

Lara et al.

Rodríguez et al.
2010

España

2011

Estados Unidos

2011

Estados Unidos

2011

Irán

2012

España

2012

España
Analizan la capacidad cardiorrespiratoria y el síndrome metabólico en los bomberos.

Relacionan la percepción de la capacidad aeróbica y el $\mathrm{VO}_{2}$ máx en bomberos.

Miden la precisión de las evaluaciones de $\mathrm{VO}_{2}$ pico en los bomberos profesionales.

Trabaja sobre la salud física del bombero y como mejorar el rendimiento y prevenir lesiones $\mathrm{y}$ muertes.

Evalúan la validez y la capacidad de prueba de la función profesional de los bomberos iraníes.

Analizan la condición física en bomberos en función de la edad.

Evalúan las demandas fisiológicas de trabajo de los bomberos forestales españoles.

En la tabla 2 se describen aquellos estudios que su muestra la constituían bomberos que eran físicamente activos, su objetivo y el tamaño de la muestra.

Tabla 2

Descripción de estudios que incluyeron a bomberos físicamente activos.

\begin{tabular}{|c|c|c|c|}
\hline Autor (es) & Objetivo & Sujetos & Características \\
\hline $\begin{array}{l}\text { Mier y } \\
\text { Gibson } \\
(2004)\end{array}$ & $\begin{array}{l}\text { Aprobar un examen estandarizado } \\
\text { submáximo que utiliza el protocolo } \\
\text { Gerkin, cinta de correr para la } \\
\text { predicción del consumo máximo de } \\
\text { oxígeno }\left(\mathrm{VO}_{2} \mathrm{max}\right) \text { de los bomberos. }\end{array}$ & $\begin{array}{l}54 \text { entre } \\
\text { hombres } \\
\text { y } \\
\text { mujeres. } \\
35 \pm 11 \\
\text { años. }\end{array}$ & $\begin{array}{l}\text { Sujetos sanos, se } \\
\text { clasificaban según } \\
\text { riesgo cardiaco y los } \\
\text { que presentaban un alto } \\
\text { riesgo cardiaco se } \\
\text { excluían del estudio. }\end{array}$ \\
\hline $\begin{array}{l}\text { Rodríguez } \\
\text { al. (2007) }\end{array}$ & $\begin{array}{l}\text { Analizar la intensidad de trabajo y el } \\
\text { esfuerzo desarrollado por el Personal } \\
\text { Especialista en Extinción de } \\
\text { Incendios Forestales (P.E.E.I.F.) } \\
\text { durante el desempeño de su trabajo } \\
\text { como aspecto fundamental abordar } \\
\text { mejoras en las medidas de }\end{array}$ & $\begin{array}{c}200 \\
\text { sujetos. } \\
27 \pm 1 \\
\text { años }\end{array}$ & $\begin{array}{l}\text { Promedios: } \\
75,20 \pm 0,80 \text { Kg } \mathrm{y} \\
174,20 \pm 0,60 \text { cm., } \\
\text { pertenecientes a } 4 \\
\text { brigadas de refuerzo } \\
\text { de incendios forestales } \\
\text { (BRIF) con una }\end{array}$ \\
\hline
\end{tabular}


prevención de riesgos laborales.

Saborit et al. Conocer la capacidad aeróbica real (2010)

(CAR) de una muestra de bomberos basada en su $\mathrm{VO}_{2}$ máx y compararla con dos medidas auto informadas: la valoración subjetiva de su capacidad aeróbica (VSCA) y la valoración del ejercicio físico realizado (VEF).

Drew-Nord et Medir la precisión de las al. (2011) evaluaciones del $\mathrm{VO}_{2}$ en bomberos

Smith, (2011)

D Analizar los factores involucrados en la salud física de los bomberos

Rodríguez et Analizar los efectos fisiológicos, al. (2012) demandas y la tensión térmica de los bomberos. experiencia de $3,20 \pm 0,30$ años en las labores de extinción de incendios.

37 IMC: $23,06 \pm 4,00$ y un bomberos porcentaje graso de varones. $15,20 \pm 2,10 \%$. $29,00 \pm$ Antigüedad mínima de 3,60 años dos años y buena capacidad física.

83 Haber aprobado el test bomberos Wellness Fitness varones Initiative (WFI) previamente con un $\mathrm{VO}_{2}$ pico de 35 $\mathrm{ml} / \mathrm{kg} / \mathrm{min}$

$40 \quad$ Físicamente activos bomberos

200 Edad promedio: 25,20 bomberos $\pm 0,40$ años, quienes forestales aprobaron las pruebas físicas de selección

Lara et al. Describir las características de (2012) composición corporal y de condición física en dos grupos de bomberos activos.

En la tabla 3 se describen aquellos estudios que realizaron sus investigaciones en poblaciones de bomberos que eran sedentarias y a su vez presentaban problemas de salud especialmente obesidad y acumulación del porcentaje de grasa. 
Tabla 3

Descripción de estudios que incluyeron a bomberos sedentarios o con problemas de salud.

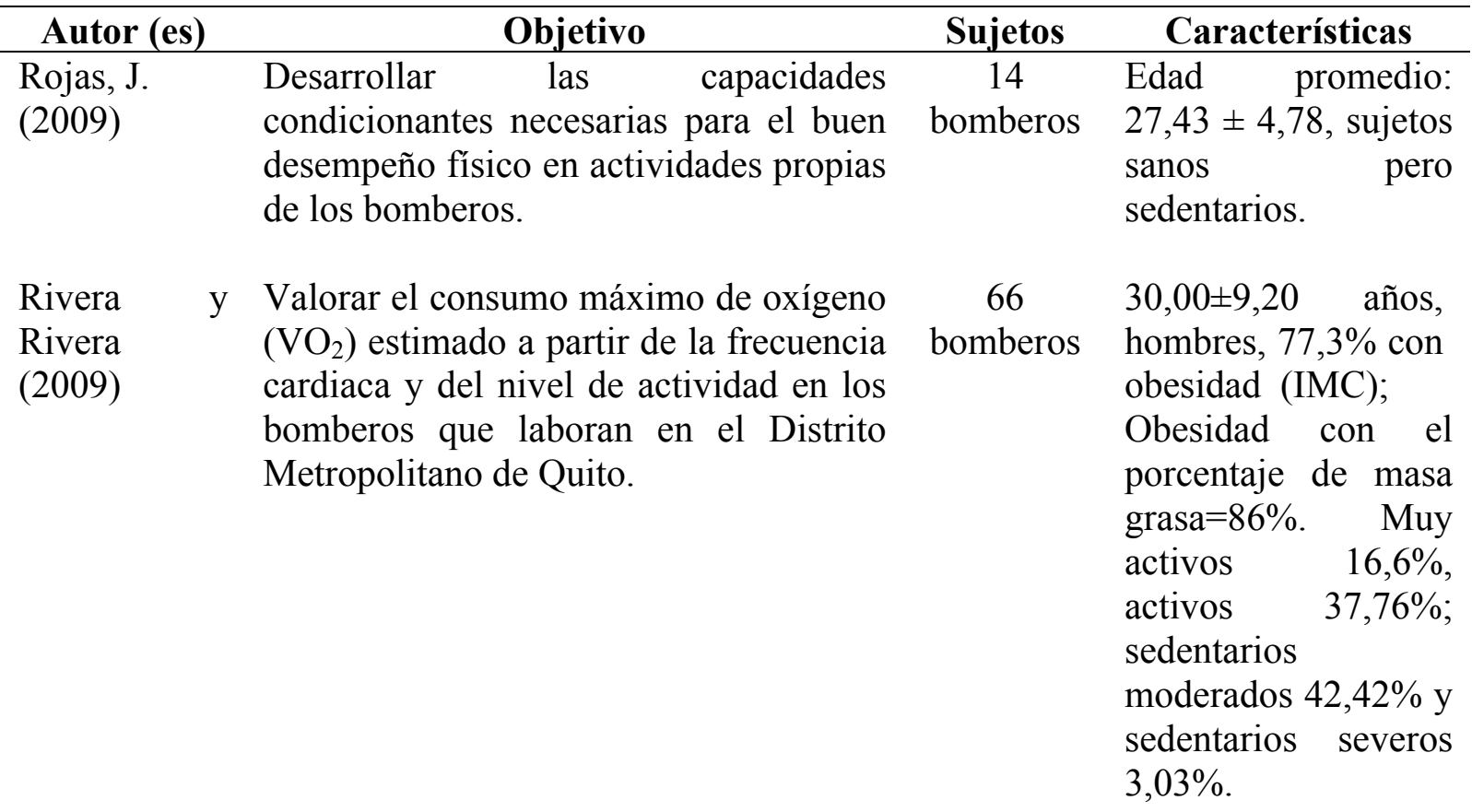

Donovan et Documentar los niveles de capacidad

214 Según el IMC: $25 \%$ al. (2009) cardiorrespiratoria y el síndrome metabólico, así como determinar si existe una relación entre estas variables, en los bomberos profesionales en los bomberos normal, $56 \%$ masculino sobrepeso, $\quad 19 \%$ EE.UU. obesidad.

muestra la componían bomberos sanos $\mathrm{y}$ bomberos con síndrome metabólico.

Existen otros estudios que no especifican si su muestra era compuesta por bomberos que se encuentran físicamente activos o no, dentro de estos estudios se trató de validar un trabajo específico de evaluación física del rendimiento en el trabajo que representa a los bomberos, se analizó diferentes pruebas de aptitud física como criterio de selección del personal, la intensidad de trabajo, el esfuerzo durante el desempeño de su trabajo y evaluar la exactitud y la capacidad de las pruebas de bomberos en proporción a su estado cardiovascular. (Mendenhall et al., 2005; Satué et al., 2007; Villa et al., 2009; Kianmehr y Nazem, 2011) (Ver tabla 4) 
URL www.una.ac.cr/mhsalud

Tabla 4

Descripción de estudios que incluyeron a bomberos sin definir su estado físico.

\begin{tabular}{|c|c|c|c|}
\hline Autor (es) & Objetivo & Sujetos & Características \\
\hline $\begin{array}{l}\text { Mendenhall } \\
\text { et al. } \\
(2005)\end{array}$ & $\begin{array}{l}\text { Validar un trabajo específico de } \\
\text { evaluación física del rendimiento en el } \\
\text { trabajo que representa a los bomberos }\end{array}$ & $\begin{array}{c}75 \\
\text { bomberos }\end{array}$ & $\begin{array}{l}\text { Rango de edad: } \\
25-45 \text { años }\end{array}$ \\
\hline $\begin{array}{l}\text { Satué et al. } \\
(2007)\end{array}$ & $\begin{array}{l}\text { Analizar el grado de fiabilidad y nivel de } \\
\text { especificidad de diferentes pruebas de } \\
\text { aptitud física utilizadas como criterio de } \\
\text { selección del personal especialista en } \\
\text { extinción de incendios forestales, tales } \\
\text { como el test de Course Navette (batería } \\
\text { I.N.F.O.C.A.), Prueba del Banco y Pack } \\
\text { Test (U.S.D.A,1991). }\end{array}$ & $\begin{array}{c}1500 \\
\text { bomberos }\end{array}$ & $\begin{array}{l}\text { Edad promedio: } \\
36,14 \pm 0,38 \text { años }\end{array}$ \\
\hline $\begin{array}{l}\text { Villa et al. } \\
(2009)\end{array}$ & $\begin{array}{l}\text { Analizar la intensidad de trabajo y el } \\
\text { esfuerzo desarrollado por el P.E.E.I.F. } \\
\text { durante el desempeño de su trabajo como } \\
\text { aspecto fundamental abordar mejoras en } \\
\text { las medidas de prevención de riesgos } \\
\text { laborales. }\end{array}$ & $\begin{array}{c}200 \\
\text { sujetos }\end{array}$ & $\begin{array}{l}\text { Edad promedio: } \\
27,00 \pm 1,00 \text { años }\end{array}$ \\
\hline $\begin{array}{l}\text { Kianmehr y } \\
\text { Nazem } \\
(2011)\end{array}$ & $\begin{array}{l}\text { Evaluar la exactitud y la capacidad de las } \\
\text { pruebas de bomberos iraníes masculinos } \\
\text { funcionales en proporción a su estado } \\
\text { cardiovascular. }\end{array}$ & $\begin{array}{c}90 \\
\text { bomberos }\end{array}$ & $\begin{array}{l}\text { Rango de edad: } \\
23-36 \text { años }\end{array}$ \\
\hline
\end{tabular}

Los diferentes estudios utilizaron una variedad de métodos para medir el $\mathrm{VO}_{2} \mathrm{max}$, Mier y Gibson (2004) utilizaron un protocolo en banda sin fin para medir el $\mathrm{VO}_{2} \mathrm{max}$, llamado protocolo de cinta de Gerkin; en el cual la intensidad se ajusta a cada minuto alternando un aumento de 0,5 millas por hora, con un grado de inclinación de un $2 \%$, el protocolo llega a su fin cuando el sujeto llega a un ritmo cardíaco igual o mayor a un $85 \%$ de la frecuencia cardiaca máxima (FCmáx).

Lara et al. (2012) realizaron la prueba de Course Navette para estimar el $\mathrm{VO}_{2}$ máx de cada sujeto, de todas las variables evaluadas de la condición física, estos autores consideran que el $\mathrm{VO}_{2}$ máx es la variable más determinante a la hora de valorar el estado físico en poblaciones que desarrollan una actividad intensa, como pueden ser bomberos o deportistas.

Por otra parte, Rodríguez et al. (2007) utilizaron un test incremental sobre un tapiz rodante; el test se inició a una velocidad de $2,7 \mathrm{~km} \cdot \mathrm{h}-1$ con una pendiente del $10 \%$; cada 3 min tanto la velocidad como la pendiente fueron modificadas hasta que el sujeto no pudo mantener la velocidad fijada. 
Saborit et al. (2010) realizaron pruebas de esfuerzo hasta la extenuación en una cinta sin fin, midiendo la frecuencia cardíaca y el $\mathrm{VO}_{2}$ máx; concluyendo que $43 \mathrm{ml} / \mathrm{kg} / \mathrm{min}$ es el valor mínimo aconsejado para desarrollar el trabajo de bombero eficazmente. Por otra parte, Lara et al. (2012) recomendaron que los valores mínimos de $\mathrm{VO}_{2}$ máx deben ser de 41,5 a $42 \mathrm{ml} / \mathrm{Kg} / \mathrm{min}$. Ambos estudios plantean cifras de $\mathrm{VO}_{2}$ max muy similares para desempeñarse como bombero; sin embargo aún faltan evidencias científicas que comprueben estos valores lo que fomenta a realizar futuros estudios que permitan establecer el rango de valores mínimos necesarios en $\mathrm{VO}_{2}$ como parámetros para el trabajo de bomberos.

En la práctica, los valores de $\mathrm{VO}_{2} \max$ son directamente proporcionales con la frecuencia cardiaca, por ende se puede utilizar la $\mathrm{FC}$ para estimar el $\mathrm{VO}_{2} \mathrm{max}$. Rodríguez et al., (2012) reportan que la $\mathrm{FC}$ media obtenida en los incendios fue de $117 \pm 2$ pulsaciones por minuto (ppm), lo que supuso a los sujetos una intensidad del 60,6 1 1,1\% de la FC máxima.

En el trabajo de Rodríguez et al. (2012) se analizó 79 incendios en los que actuaron los bomberos estudiados, la duración media de los incendios forestales fue de 225,3 $\pm 6,2$ min. La FC máxima alcanzada en los incendios fue de $169 \pm 1 \mathrm{ppm}$. Teniendo en cuenta los valores máximos alcanzados en la prueba de esfuerzo realizada la intensidad de trabajo que realizaron los sujetos en los incendios fue del 60,6 1 ,1\% de la FC máxima.

En el proceso de preparación física de los bomberos en inducción en Costa Rica; se ejecutó un mesociclo de tres meses con catorce participantes, la variedad de experiencia deportiva era amplia, estaban aquellos que alguna vez realizaron algún tipo de actividad física y otros que no contaban con experiencia en la práctica de actividad física, de forma general ninguno se había sometido anteriormente a la aplicación de los principios de entrenamiento deportivo, realizando entrenamientos sin ninguna base científica; la edad promedio de los sujetos participantes en este proceso de inducción fue de $27,43 \pm 4,78$ años y el $\mathrm{VO}_{2}$ max en promedio fue de $45,45 \pm 5,50 \mathrm{ml} / \mathrm{kg} / \mathrm{min}$, utilizando la prueba Cooper para determinar el mismo (Rojas, 2009).

Tabla 6

Consumo máximo de oxígeno $\left(\mathrm{VO}_{2} \mathrm{max}\right)$ en $\mathrm{ml} / \mathrm{kg} / \mathrm{min}$ obtenido en los estudios analizados en la revisión sistemática.

\begin{tabular}{lccl}
\hline \multicolumn{1}{c}{ Autor } & Año & $\begin{array}{c}\text { VO2max } \\
\text { (ml/kg/min) }\end{array}$ & \multicolumn{1}{c}{ Características } \\
\hline Gaskill et al. & 2003 & 49,00 & Bomberos sanos, hombres \\
Mier y Gibson & 2004 & $49,80 \pm 8,30$ & Bomberos sanos, hombres y mujeres \\
Lawson et al. & 2004 & 58,21 & Bomberos sanos \\
Mendenhall et al. & 2005 & 40,00 & Bomberos sanos, hombres \\
Sharkey & 2007 & 50,00 & Bomberos sanos \\
(cit. por Rodríguez et al) & 2007 & $56,70 \pm 1,90$ & Bomberos sanos \\
Rodríguez et al. & 2007 & $42,40 \pm 4,40$ & Hombres, activos \\
Dreger y Petersen & & $39,20 \pm 5,60$ & Combinado, activos \\
& & &
\end{tabular}




\begin{tabular}{lccl} 
Satué et al. & 2007 & $48,41 \pm 0,59$ & Bomberos sanos \\
Espinosa et al. & 2007 & $54,50 \pm 20$ & Bomberos sanos \\
Donovan et al. & 2009 & 46,60 & Combinado, hombres \\
& & $47 \pm 60$ & Bomberos sanos \\
& & $42 \pm 50$ & Bomberos con síndrome metabólico \\
Villa et al. & 2009 & 42,00 & Bomberos sanos \\
Rojas, J. & 2009 & $45,45 \pm 5,50$ & Bomberos sanos, sedentarios \\
Rivera y Rivera & 2009 & 53,74 & Bomberos sedentarios y obesos \\
Saborit et al & 2010 & 43,00 & Bomberos sanos, hombres \\
Drew-Nord et al. & 2011 & 49,00 & Bomberos sanos, hombres \\
Smith, D. & 2011 & 56,00 & Bomberos sanos y activos \\
Kianmehr y Nazem & 2011 & $47,53 \pm 3,74$ & Bomberos sanos \\
Lara et al. & 2012 & 47,26 & Bomberos sanos \\
Rodríguez et al. & 2012 & $56,20 \pm 0,90$ & Bomberos sanos \\
\hline
\end{tabular}

Nótese en la tabla 6 que el rango de los datos del $\mathrm{VO}_{2} \mathrm{max}$ en los diferentes estudios realizados en los bomberos va desde los $39,20 \mathrm{ml} / \mathrm{kg} / \mathrm{min}$ hasta 10 s $58,21 \mathrm{ml} / \mathrm{kg} / \mathrm{min}$; muchos de estos datos son promedios determinados al realizar los respectivos análisis estadísticos en cada uno de los estudios. Wilmore y Costill (2007) establecen como parámetros normales para los no deportistas para un rango de edad de 20 a 29 años, muy similar a la mayoría de estudios analizados en esta revisión, en hombres un $\mathrm{VO}_{2} \mathrm{max}$ normal de 43 a $52 \mathrm{ml} / \mathrm{kg} / \mathrm{min}$ y en mujeres de este rango de edad de 33 a $42 \mathrm{ml} / \mathrm{kg} / \mathrm{min}$. En los estudios analizados no se hace diferencia según el género, pero en su gran mayoría las muestras están compuestas por hombres, por lo cual el rango de $\mathrm{VO}_{2}$ max propuesto por Wilmore y Costill (2007) es muy similar al rango de datos obtenidos (43 a 58,21 $\mathrm{ml} / \mathrm{Kg} / \mathrm{min}$ ) en los diversos estudios analizados en esta revisión sistemática. Sin embargo, una debilidad de estos estudios consiste en no distinguir entre hombres y mujeres, además de la falta de control del nivel de actividad física cotidiana de los bomberos, fuera de su trabajo.

Es fundamental considerar que después de los 25 años, se presenta una disminución de un $10 \%$ del $\mathrm{VO}_{2}$ max por cada década que pasa, tanto para hombres como para mujeres, esto indiferentemente del nivel de actividad física; por ello en un rango de edad entre 30 y 39 años, el $\mathrm{VO}_{2} \max$ se encuentra entre 39 a $48 \mathrm{ml} / \mathrm{kg} / \mathrm{min}$ en varones y de 30 a $38 \mathrm{ml} / \mathrm{kg} / \mathrm{min}$ en mujeres; datos inferiores a los rangos entre 20 y 29 años (Wilmore y Costill, 2007), y que se debe considerar al realizar un análisis del $\mathrm{VO}_{2} \max$ en los bomberos.

\section{CONCLUSIONES}

El determinar el $\mathrm{VO}_{2} \max$ oxígeno en los bomberos permite no sólo identificar problemas en la salud que puedan estar afectando a los sujetos, sino, también convertirse en una herramienta efectiva y muy estudiada como mecanismo en el control de los entrenamientos, específicamente en los procesos de preparación física, tanto para el desarrollo de las cargas de entrenamiento, como a su vez para establecer los sistemas de evaluación constante que se deben ejecutar para ejercer una profesión como esta. 
En general se puede determinar con base a los resultados de los estudios que las diferentes poblaciones de bomberos, indiferentemente del lugar de origen de la muestra estudiada en las investigaciones revisadas tienen una buena o aceptable capacidad aeróbica, presentando valores de $\mathrm{VO}_{2}$ max por encima de $\operatorname{los} 43 \mathrm{ml} / \mathrm{kg} / \mathrm{min}$, mínimo recomendable para al menos poseer una buena capacidad aeróbica.

Por otra parte una deficiencia aeróbica es sin duda un factor de riesgo a padecer de enfermedades cardiovasculares como consecuencia del humo y de las sustancias tóxicas, a las que muchas veces se exponen los bomberos.

Es fundamental para futuras investigaciones no sólo el medir el $\mathrm{VO}_{2} \mathrm{max}$, sino, también crear protocolos específicos para esta población, que puedan evidenciarse científicamente en cuanto su efectividad y así ser aplicados a las poblaciones de bomberos.

\section{REFERENCIAS}

Ares, A. (2008). Bomberos: cómo enfocar la seguridad y salud en una profesión de especial riesgo. Gestión Práctica de Riesgos Laborales, 50, 24.

Berninger, A.; Webber, M.; Weakley, J.; Gustave, J.; Zeig-Owens, R.; Lee, R.; Al-Othman, F.; Cohen, H.; Kelly, K. y Prezant, D. (2010). Quality of life in relation to upper and lower respiratory conditions among retired 9/11-exposed firefighters with pulmonary disability. Qual Life Res, 19:1467-1476

Donovan, R.; Nelson, T.; Peel, J.; Lipsey, T.; Voyles, W. y Gay Israel, R. (2009). Cardiorespiratory fitness and the metabolic syndrome in firefighters. Occupational Medicine; 59:487-492.

Drew-Nord, D., Myers, J.; Nord, S.; Oka, R; Hong, O. y Froelicher, E. (2011). Accuracy of peak VO2 assessments in career firefighters. Journal of Occupational Medicine and Toxicology, (6) 25.

Dreger, R. y Petersen, S. (2007). Oxygen cost of the CF-DND fire fit test in males and females. Appl. Physiol. Nutr. Metab. (32) 454-462

Espinosa, V.; Roca, E.; Drobnic, F.; Prat, R.; Mirallas, J. y Barbany, J. (2007). Suplementación con glicerina para prevenir la deshidratación en tareas simuladas de bomberos. Archivos de Medicina del Deporte. Facultad de Medicina, Universidad Barcelona, España. 
Gaskill, S., Ruby, B., Heil, D., Sharkey, B., Slivka, D., y Lankford, E. (2003). Seasonal changes in wildland firefighters aerobic fitness. Medicine Science in Sports Exercise. 35(5), 131.

Henderson, N.; Berry, M.; Matic, T. (2007). Field measures of strength and fitness predict firefighter performance on physically demanding tasks. Personnel psychology, (60) $431-473$.

Kianmehr, P., \& Nazem, F. (2011). Evaluation of validity and capability of professional function test of Iranian firemen. (English). Journal Of Military Medicine, 13(3), 147-153.

Lara, A.; García, J.; Torres-Luque, G. y Zagalaz, M. (2012). Análisis de la condición física en bomberos en función de la edad. Apunts Med Esport, 2(1) 30-37.

Lawson, L., Crown, E., Ackerman, M., y Dale, J. (2004). Moisture effects in heat transfer through clothing systems for wildland firefighters. International Journal of Occupational safety and Ergonomics. 10: 227-238.

López, J. y Fernández, A. (2006). Fisiología del ejercicio. (3 ed). Panamericana, España.

Mbanu, G.; Wellenius, M.; Mittleman, L.; Stallings, L. y Kales, S. (2007). Seasonality and coronary heart disease deaths in United States firefighters. Chronobiology International, 24(4): 715-726.

Mendenhall, D.; Moffatt, S.; Williams, T.; Reeves, M.; Greeson, J.; Shelton, C.; Stahl, H.; Zollinger, T.; Saywell, R. y Muegge, C. (2005). Validation of a physical work performance evaluation for incumbent firefighters. Fire Engineering, United States.

Mier, C. y Gibson, A. (2004). Evaluation of a treadmill test for predicting the aerobic capacity of firefighters. Occupational Medicine; 54:373-378.

Rivera, M. y Rivera, H. (2009). Estudio del Consumo máximo de oxígeno, sedentarismo y carga de trabajo físico en tres Compañías de Bomberos del Distrito Metropolitano de la ciudad de Quito, Ecuador. Proceedings of the Seventh international Conference on Occupational Risk Prevention. 
Rodríguez, J.; Villa, J.; López, J.; Moreno, S.; Pernía, R.; Ávila, M.; García, J. y Mendonça, P. (2007). Análisis de la intensidad de esfuerzo alcanzada por el personal especialista en extinción de incendios forestales (PEEIF). Universidad de León, España.

Rodríguez, J.; López, J.; Pernia, R.; Carballo, B.; García, J.; Foster, C. y Villa, J. (2012). Physiological work demands of Spanish wildland firefighters during wildfire suppression. Int Arch Occup Environ Health . 85:221-228

Rojas, J. (2009). Informe final del proceso de preparación física de los bomberos en inducción en Cartago. Instituto Nacional de Seguros de Costa Rica. Manuscrito no publicado.

Saborit, J.; Valle, M.; Montoliú, M.; Martínez, P.; Nistal, P. y González, V. (2010). Relación entre la percepción de la capacidad aeróbica y el VO2máx en bomberos. Psicothema, 131-136.

Satué, J.; Villa, J.; Rodríguez, J.; García, J; Moreno, S; Ávila, C y Pernía, R. (2007) Estudio de los factores condicionantes del rendimiento físico del Personal Especialista en la Extinción de Incendios Forestales: pruebas de aptitud física de selección de personal. Wilfire, Sevilla, España.

Smith, D. (2011) Firefighter Fitness: Improving Performance and Preventing Injuries and Fatalities. Current Sports Medicine Reports, 10(3), 167-172.

Villa, J.; López, J.; Ávila, M.; Rodríguez, J.; Pernía, R.; García, J.; Moreno, S.; Marqués, R. y Mendonça, P. (2009). Monitorización de la frecuencia cardiaca en las labores de extinción de incendios forestales, para la prevención de riesgos laborales. Prevención: Revista técnica de seguridad y salud laborales. 181: 6-26.

Wilmore, J. y Costill, D. (2007) Fisiología del esfuerzo y del deporte. PAIDOTRIBO, España.

Fecha de recepción: 3 de diciembre de 2012.

Fecha de aceptación: 3 de mayo de 2013.

Fecha de publicación: 31 de julio de 2013. 